\title{
Clinical Evaluation of Intense Pulsed Light vs. Combined Treatment of Intense Pulsed Light and NDYAG Laser for Facial Rejuvenation in Latin American Women
}

\author{
Aura Ruiz ${ }^{1-4 *}$ and Martha Rivero ${ }^{4,5}$ \\ ${ }^{1}$ Aesthetic Medicine Magister, University of the Balearic Islands, Spain \\ ${ }^{2}$ Dermatology Teacher, National University of Colombia, Bogotá, Colombia \\ ${ }^{3}$ Science Director at Laser Medical Care Institute, Bogotá, Colombia \\ ${ }^{4}$ Member ASMLS, USA \\ ${ }^{5}$ Aesthetic Medicine specialist Doctor, University of Rosario, Bogotá, Colombia
}

\begin{abstract}
Background and Objective: The aim of this study was to compare the effectiveness of two non-ablative techniques for facial rejuvenation in Latin-American skin types.

Materials and Methods: 36 consecutive patients with moderate-to-advanced photoaging were treated with IPL or a combination of IPL/Nd YAG laser. Clinical improvements were assessed at baseline and 30 days after two treatments. The results were compared with a non- parametric statistical test. Side effects were reported.

Results: 18 patients received treatment with IPL and 18 patients with combination IPL/NdYAG laser. Significant differences between baseline and 30 days post- treatments were observed for all features. Outcomes achieved with IPL/NdYAG were significantly higher. Side effects were limited to mild transitory erythema.

Conclusions: Non-ablative techniques with IPL or combination IPL/NdYAG laser long pulse for facial rejuvenation are safe and effective treatments in Latin- American skin types with almost no side effects. Outcomes achieved with IPL/NdYAG laser were higher than using IPL alone in this study.
\end{abstract}

Keywords: Skin rejuvenation; Photo rejuvenation; IPL; Intense pulsed light; No ablative treatment; Nd YAG lasers

\section{Introduction}

The main objective of non-ablative rejuvenation is to aesthetically enhance the features that are related to photoaging of the skin, including the appearance depigmentation, state of fine wrinkles, thick texture, prominent pores, telangiectasias, and laxity of the skin [15]. By definition, in non-ablative methods, there is no epidermal damage, therefore less downtime and successful results, being highly recommended, especially in darker phototype skins [6]. Several studies compare different laser techniques and results in photo facial rejuvenation techniques. The Nd:YAG and IPL and their combination can be pointed as useful, which has been demonstrated histologically [5,79]. Other studies evaluate clinical outcomes through photographic monitoring $[1,2,10,11]$

The melanin absorption coefficient decreases exponentially as the wavelength increases. Near infrared and infrared wavelengths can be used effectively for facial rejuvenation in darker skin types. $1.064 \mathrm{~nm}$ is safer for darker skin [7-10]. Epidermal cooling is essential when treating dark skin with visible and near-infrared lasers and light devices. Without efficient cooling, the light absorbed by melanin is converted to heat, creating unwanted thermal injury [6,7]. The use of antioxidants such as lipoic acid and phytoestrogens in skin protection has been proven to have an effect on the UV rays and also reduce oxidative stress, improving its quality (Article of lipoic acid). Associated with laser techniques compound preparations with retinoic acid, hydroquinone and alpha-hydroxy acids, etc., [8], improve and maintain the result in the process of long-term facial rejuvenation, also diminish the effects of post inflammatory hyperpigmentation.

The dark skin types are associated with a high risk of complications after laser procedures. The non-ablative facial skin rejuvenation [6-8] has not been extensively evaluated in Latin American skin types.

\section{Materials and Methods}

We performed a retrospective and descriptive study on the dermoaesthetic characteristics of a group of female patients attending the Laser Medical Care (Aesthetic Medicine and Laser center) between 2008 and 2009 , motivated by the need to improve their facial appearance. The study included a group of 36 patients who were chosen through the center's medical records and who had completed photographic records necessary for the evaluation. The techniques used at the time were chosen according to the discretion of the treating physician.

We selected two groups of equal number of patients (18 each) the first group underwent the technique of Intense Pulsed Light IPL and the second group underwent a technique that combined intense pulsed light and Nd: YAG Laser with Synchro platform HP from DEKA. Following parameters were used, IPL 550-950 nm: spot of $18 \mathrm{~mm} \times 46$ $\mathrm{mm}$, with a fluency of $7.5-11 \mathrm{~J} / \mathrm{cm}^{2}, 3$ pulses, each pulse duration 5 msec, $110 \mathrm{msec}$ delay, 2 passes. $1064 \mathrm{~nm} \mathrm{Nd:} \mathrm{YAG} \mathrm{laser} \mathrm{pulse} \mathrm{length:}$ of $15 \mathrm{~mm}$ spot, with a fluency of $52 \mathrm{~J} / \mathrm{cm} \mathrm{2,3}$ pulses, pulse duration $15 \mathrm{msec}, 125 \mathrm{msec}$ delay, frequency $1 \mathrm{~Hz}, 4$ passes. For the second combined treatment group the technical procedure was performed with the IPL and then Nd YAG laser.

${ }^{*}$ Corresponding author: Aura Ruiz M.D, Aesthetic Medicine Magister, University of the Balearic Islands, Spain, Tel: +5718052805; E-mail: aura.ruiz@lacare.co

Received: October 14, 2014; Accepted: October 17, 2014; Published: October 19, 2014

Citation: Ruiz A, Rivero M (2014) Clinical Evaluation of Intense Pulsed Ligh vs. Combined Treatment of Intense Pulsed Light and NDYAG Laser for Facial Rejuvenation in Latin American Women. Pigmentary Disorders 1:142. doi: 10.4172/2376-0427.1000142

Copyright: (c) 2014 Ruiz A, et al. The terms of the Creative Commons Attribution License, which permits unrestricted use, distribution, and reproduction in any medium, provided the original author and source are credited. 
In both groups the patients were exposed to cold air with Smart Cryo DEKA. 10 minutes before starting the procedure and during it. All patients also underwent treatment which consisted of a daily selfapplication during the time elapsed after the first and second treatment session with an interval of 30 days in each session as follows: "AgexSoy cream" (soy isoflavone, lipoic acid) an application in the morning accompanied by a sun protector with a screen larger than 30 . At night an application of a compound preparation based on $3 \%$ hydroquinone, retinoic acid $0.025 \%$ and hydrocortisone $1 \%$.

The evaluation criteria were the same for both groups: fine lines, deep wrinkles, mottled pigmentation, telangiectasia, laxity and overall appearance. The criteria of evaluation were not categorized. All patients were evaluated by two physicians specializing in aesthetic medicine and with extensive experience in dermo-aesthetic treatments through the assessment of digital photographic records, ignoring the relationship between each patient and the technique used in them. We used a standardized rating scale for each of the criteria. Rating scales for each categorical criterion were converted to percentage scales which allowed turning them into continuous variables.

The study was initiative of the "LACARE Laser medicine Institute" team, seeking to organize the records of patients treated in the center and evaluating some criteria in them that allow showing results to guide the effectiveness of treatments and techniques used. It was posed the report of aspects or unwanted complications during the application of techniques.

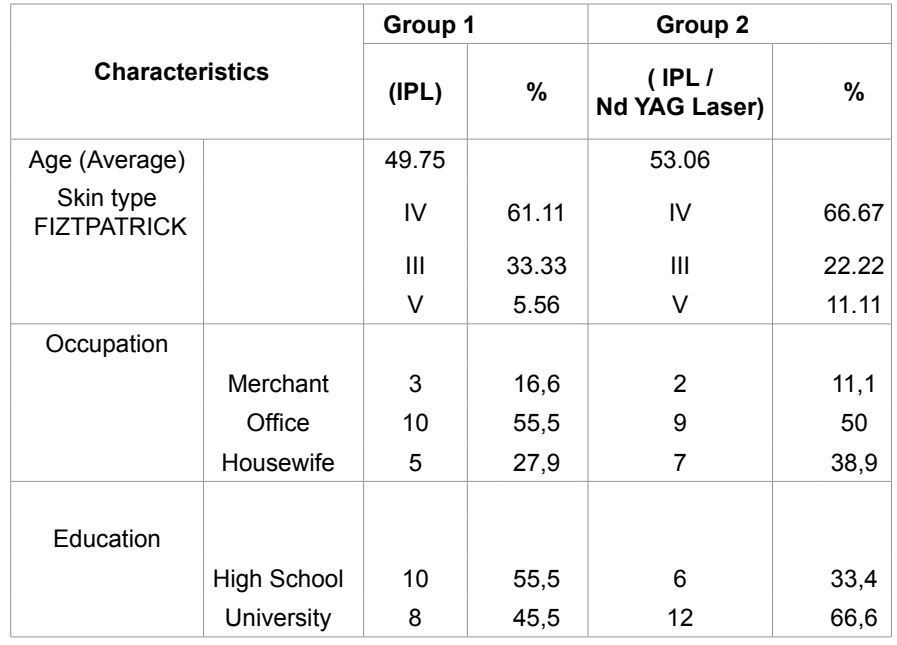

Table 1: General Characteristics of the Patients.



Figure 1: Evaluation of fine lines by treatment group.

\section{Results}

We evaluated the medical records and photographs of 36 patients, which in turn corresponded to $7.7 \%$ of all patients treated at the Centre and who consulted voluntarily to enhance facial features.18 patients were treated with IPL (group 1) and 18 with a combination of IPL and laser NdYag (group 2). The general characteristics of the patients evaluated in each group are recorded in Table 1.

The average age for group 2 is higher in comparison to the average for patients in group 1. The standard deviations for each group were 7.91 and 7.15 respectively, this variable is important if we take into account that it may be a factor in the effectiveness of treatments. The skin photo type for both groups is type IV over $60 \%$ which corresponds to women with brown skin color characteristic of our Latin-American women. The occupation of the women is a variable that determines the exposure to environmental factors that influence the outcome of the treatments evaluated. We found that for group $1(83.4 \%)$ and group $2(88.9 \%)$ of the women studied had occupations (office, housewives) that by their nature do not expose patients so often to these factors, which we believe does not influence the outcome of the treatments used.

Finally, as a general characteristic, we studied the level of education as a factor that is closely related to creating awareness in higher education regarding the care and adherence to the treatment used. We found that group 2 had a higher percentage of women with university educational level (66.6\%) compared to group $1(45.5 \%)$ which could be a factor influencing better results in group 2, but the features and design of this study did not allow us to test this hypothesis .

The evaluation in categorical variables of the criteria analyzed in each group was converted to continuous variables by averaging the ratings of the two evaluators. It was performed the nonparametric Mann-Whitney U-test to compare two independent samples, observing a statistically significant difference between the treatment groups evaluated, being better the result acquired with the intervention performed in group 2 .

The following charts show the results for each criterion evaluated:

In this criterion, it was evaluated the decrease of fine lines as an influential factor in the overall appearance of the skin, finding that in the second group the average of positive responses was over $50 \%$ compared to group 1 which showed a result of $25 \%$, it was identified in the latter group between the responses a data edge in the described graph (Figure 1).

The decrease in the depth of wrinkles is the aspect to evaluate in this criterion, which according to the weighted results described in Figure 2 , show a satisfactory result in the second group with $38 \%$ compared to the first group. In a similar vein, the data identified so far in group 1 is consistent with the evaluation of the above criteria (Figure 2).

The differences found in the evaluation of this criterion are not very significant, taking into account the responses from the criteria evaluated before, but the trend of a better outcome continues in the second group with an assessment rate higher than $50 \%$, with relation to the other group which was $38 \%$. The aspect to evaluate in this criterion is the degree of reduction of pigmentation observed in the skin of the patient (Figure 3).

Among the most influential criteria in the evaluation of the techniques implemented, we observed the degree of reduction in redness or telangiectasias presented in the patients. Figure 4 shows that in general, this criterion did not obtain a good result by the observers in relation to the above criteria, but a better average percentile score 


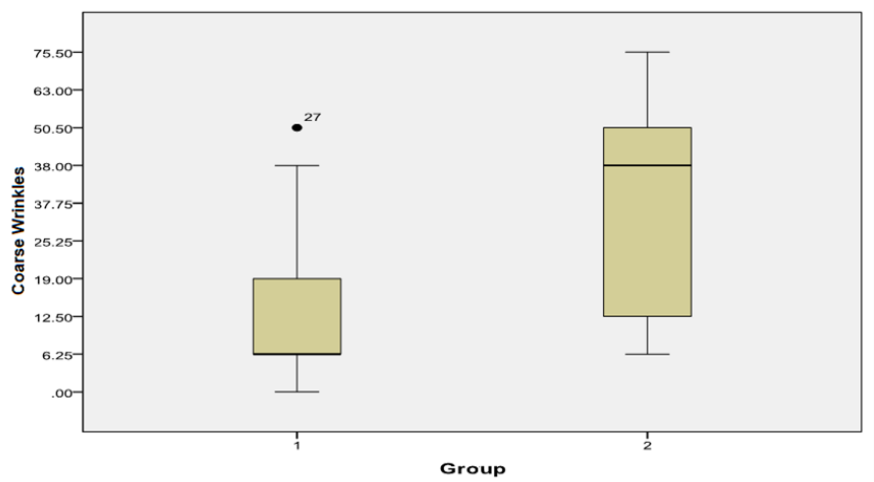

Figure 2: Evaluation of Coarse Wrinkles by treatment group.

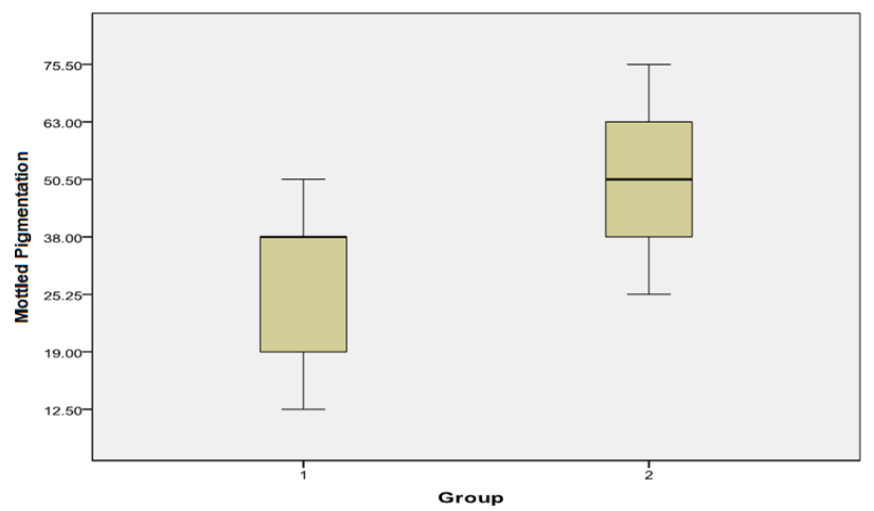

Figure 3: Evaluation of Mottled Pigmentation according to type of treatment.

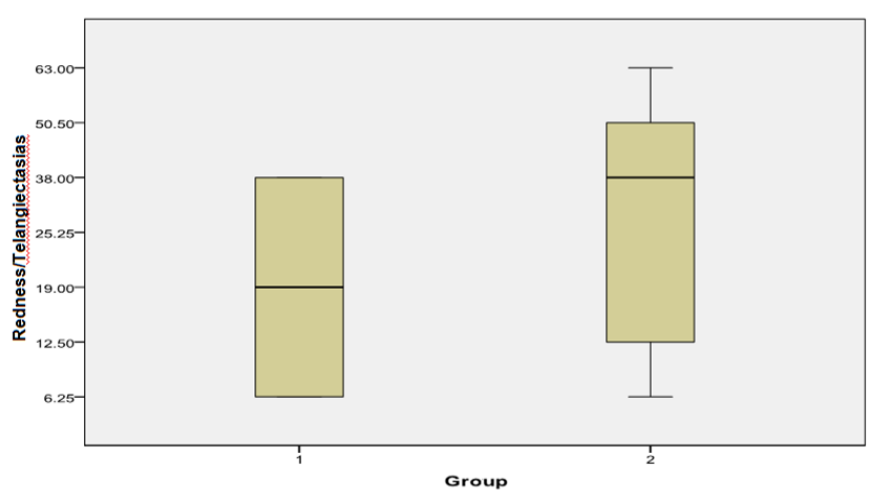

Figure 4: Evaluation of redness/telangiectasias.

persisted in the second group with $38 \%$ compared to the first group which was $19 \%$ (Figure 4).

The degree of improvement of laxity is evaluated taking into account its decrease as a factor that favors facial rejuvenation, average results presented for both groups and described in the Figure 5 shows a result consistent with the criteria described above, with a best result for the second group of $38 \%$ compared to the first group was 19\% (Figure 5).

The overall aesthetic improvement or overall appearance is the aspect which combines the criteria evaluated before. It allows concluding the effectiveness of the techniques evaluated in each patient group, confirming the results described in the previous graphs, the Figure 6 shows that the second group showed a result above 50\% compared to $25 \%$ in the first group. It is important to highlight the edge data of the second group described in the chart (Figure 6).

Photographies at baseline and 30 days after second combined treatment with IPL/NdYAG (Figure 7).

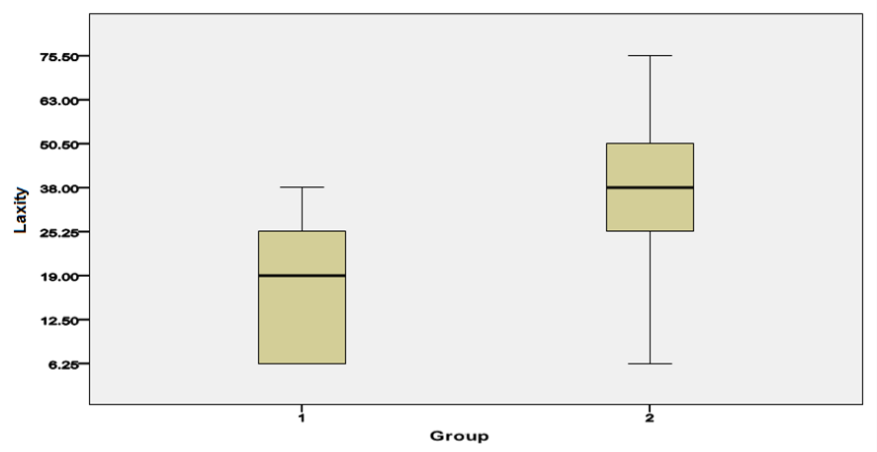

Figure 5: Assessment of laxity by type of treatment.

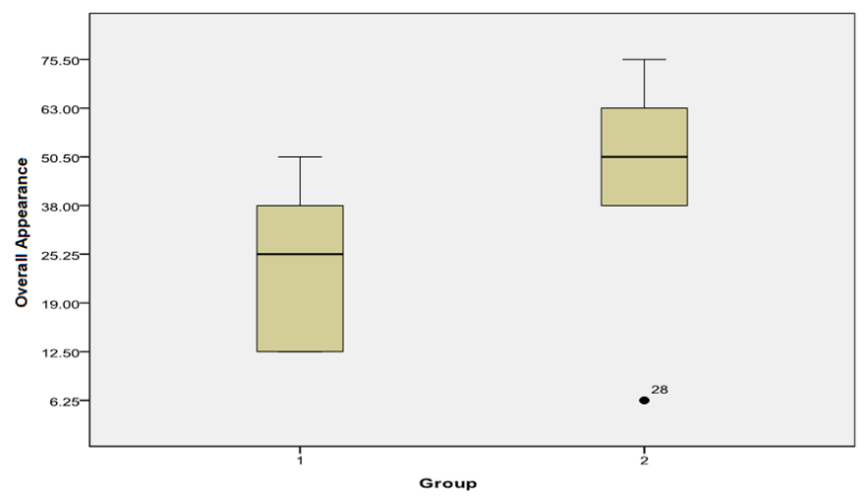

Figure 6: Evaluation of overall appearance according to type of treatment.

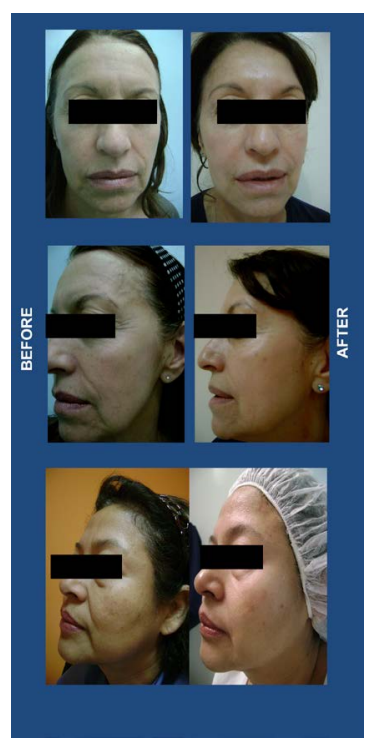

Figure 7: Clinical images 


\section{Discussion}

Non-ablative photo rejuvenation is the one in which there is a dermal change, specifically a stimulation of collagen production without causing epidermal injury, resulting in less disability time and less postoperative discomfort [6]. Among the non-ablative rejuvenation methods, it is possible to find light devices, pulsed light or IPL and different types of lasers. The IPL and the Nd:YAG were the ones evaluated in this study with positive results for skin phototype characteristic of Latin American women.

Importantly, there is no published literature describing the effectiveness of the techniques evaluated in this study IPL, Nd:YAG Laser in Latin American population, taking into account that the characteristics of skin (phototypes) are different in relation to other continents.

In the general factors specified in the study that might influence the results regarding the effectiveness of treatments, no significant differences were identified, allowing us to show that the effective treatment is the result of therapeutic characteristics in the techniques used.

The differences in each study group of skin phototypes are similar. It is described a higher percentage of phototype IV (above 60\%) characteristic of Latin American women, something that allowed us to guide the study adequately to this population, achieving the objective.

The techniques used for both groups are oriented towards achieving results in overall photo rejuvenation of the patients, but with regard to the criteria evaluated, there are efficiency differences in each of these. For example, unlike IPL, Nd: YAG laser has a higher affinity to hemoglobin, which is evident in comparison to the results obtained in the standard of "redness or telangiectasias" that although in general relation to other criteria evaluated had no significant result, patients undergoing the combined technique in this approach had a better outcome.

The results of this study reaffirm what is described in the literature about the benefits in the use of combined techniques (IPL - Nd: YAG laser) in relation to simple ones (IPL). It is important to mention that both are non-ablative techniques, when their efficacy is compared with ablative techniques, it reduces side effects in high phototypes. We believe that the combined technique is more effective; given that its physical properties potentiate the result regarding all criteria evaluated. In the case of the simple technique can be effective for some criteria but not for others.

As it is known, photo rejuvenation techniques have a predisposition to pigmentation in patients with high skin phototypes, making it necessary to use skin cosmetic treatments with depigmentation substances that counteract this effect.

Active ingredients used in this study as cosmetic prophylaxes such as soy isoflavones, vitamin $\mathrm{C}$, vitamin $\mathrm{E}$ and lecithin, have an antioxidant and sunscreen protection effect in patients [12-18]. Also, the lipoic acid is a potent antioxidant that diminishes oxidative stress and promotes collagen remodeling in patients exposed to combined treatments [16], which favored the absence of adverse effects such as pigmentation of the skin in our study.

The effectiveness of combined techniques of non-ablative photo rejuvenation with skin cosmetics treatment is more evident in patients with aging skin, due to higher photo base damage.

The epidermal changes following treatment with the combined technique IPL / Nd:YAG Laser are most evident in the short term, in relation with the remodeling of collagen and elastin that appear in a longer time period $[7,8]$, this fact was demonstrated in the evaluation performed in the second session of treatment on the 60th day, in the criteria for coarse wrinkles, laxity and fine wrinkles, favoring the latter in greater proportion in relation with other criteria evaluated.

\section{References}

1. Goldberg DJ, Samady JA (2001) Intense pulsed light and Nd:YAG laser nonablative treatment of facial rhytids. Lasers Surg Med 28: 141-144.

2. Brazil J, Owens $P(2003)$ Long-term clinical results of IPL photorejuvenation. J Cosmet Laser Ther 5: 168-174.

3. Trelles M, Allones I, Vélez M, Mordon S (2004) Nd:YAG laser combined with IPL treatment improves clinical results in non-ablative photorejuvenation. J Cosmet Laser Ther 6: 69-78.

4. Shah GM, Kilmer SL (2005) Combined nonablative rejuvenation techniques Dermatol Surg 31: 1206-1210.

5. Ross EV, Smirnov M, Pankratov M, Altshuler G (2005) Intense pulsed light and laser treatment of facial telangiectasias and dyspigmentation: some theoretical and practical comparisons. Dermatol Surg 31: 1188-1198.

6. Goldman MP, Weiss RA, Weiss MA (2005) Intense pulsed light as a nonablative approach to photoaging. Dermatol Surg 31: 1179-1187.

7. Munavalli GS, Weiss RA, Halder RM (2005) Photoaging and nonablative photorejuvenation in ethnic skin. Dermatol Surg 31: 1250-1260.

8. Rokhsar CK, Lee S, Fitzpatrick RE (2005) Review of Photorejuvenation: Devices, Cosmeceuticals, or Both? Dermatol Surg 31: 9

9. Nouri K, Rivas MP, Bouzari N, Faghih S (2006) Nonablative lasers. J Cosme Dermatol 5: 107-114.

10. Negishi K, Kushikata N, Takeuchi K, Tezuka Y, Wakamatsu S (2006) Photorejuvenation by intense pulsed light with objective measurement of skin color in Japanese patients. Dermatol Surg 32: 1380-1387.

11. Iyer S, Carranza D, Kolodney M, Macgregor D, Chipps L, et al. (2007) Evaluation of procollagen I deposition after intense pulsed light treatments at varying parameters in a porcine model. J Cosmet Laser Ther 9: 75-78.

12. Berlin AL, Dudelzak J, Hussain M, Phelps R, Goldberg DJ (2008) Evaluation of clinical, microscopic, and ultrastructural changes after treatment with a nove Q-switched Nd:YAG laser. J Cosmet Laser Ther 10: 76-79.

13. Izikson L (2008) Laser photorejuvenation of Asian and ethnic skin. J Cosmet Laser Ther 10: 161-166.

14. Fodor L, Carmi N, Fodor A, Ramon Y, Ullmann Y (2009) Intense pulsed light for skin rejuvenation, hair removal, and vascular lesions: a patient satisfaction study and review of the literature. Ann Plast Surg 62: 345-349.

15. Tanaka Y, Matsuo K, Yuzuriha S (2010) Long-term histological comparison between near-infrared irradiated skin and scar tissues. Clin Cosmet Investig Dermatol 3: 143-149.

16. Elsaie ML, Lloyd HW (2008) Latest laser and light-based advances for ethnic skin rejuvenation. Indian J Dermatol 53: 49-53.

17. Morganti $P$ (2009) The photoprotective activity of nutraceuticals. Clin Dermato 27: 166-174.

18. Draelos ZD (2008) The cosmeceutical realm. Clin Dermatol 26: 627-632. 